\title{
Compte rendu de Jean-Baptise Pompallier. Vicaire apostolique des Maoris (1838-1868), de Yannick Essertel
}

\section{Fanny Wonu Veys}

\section{(2) OpenEdition}

Édition électronique

URL : http://journals.openedition.org/jso/7512

DOI : $10.4000 /$ jso.7512

ISSN : $1760-7256$

\section{Éditeur}

Société des océanistes

Édition imprimée

Date de publication : 31 décembre 2016

Pagination : 292-294

ISSN : 0300-953x

Référence électronique

Fanny Wonu Veys, "Compte rendu de Jean-Baptise Pompallier. Vicaire apostolique des Maoris

(1838-1868), de Yannick Essertel », Journal de la Société des Océanistes [En ligne], 142-143| 2016, mis en ligne le 31 décembre 2016, consulté le 24 septembre 2020. URL : http://journals.openedition.org/ jso/7512 ; DOI : https://doi.org/10.4000/jso.7512

Ce document a été généré automatiquement le 24 septembre 2020.

(c) Tous droits réservés 


\title{
Compte rendu de Jean-Baptise Pompallier. Vicaire apostolique des Maoris (1838-1868), de Yannick Essertel
}

\author{
Fanny Wonu Veys
}

\section{RÉFÉRENCE}

ESSERTEL Yannick, 2014. Jean-Baptise Pompallier. Vicaire apostolique des Maoris (1838-1868), Paris, Les Éditions du Cerf, 448 p., bibliogr., annexes, ill. en couleur dans le texte.

1 La biographie de Jean-Baptiste Pompallier, écrite par l'historien Yannick Essertel, s'appuie sur et s'inspire de l'anthropologie sociale et historique pour reconstruire la vie de celui qui sera pendant trente ans - de 1838 à 1868 - le premier vicaire apostolique d'Océanie occidentale en Nouvelle-Zélande. L'auteur explique que le jeune JeanBaptiste, né le 12 décembre 1801, grandit dans une période postrévolutionnaire de renouveau religieux où sont fondées de diverses institutions missionnaires comme la Société des Pères maristes, organisme qui jouera un rôle essentiel dans l'évangélisation de l'Océanie. Bien que n'ayant pas prononcé ses vœux comme mariste, c'est au jeune abbé Pompallier, dynamique et animé d'un zèle de reconquête d'âmes, que revient la tâche de vicaire apostolique. Essertel argumente que Jean-Claude Colin, supérieur général de la Société de Marie, voit en lui - parmi d'autres d'ailleurs - la personne capable de porter la stratégie missionnaire qui se développera sur les niveaux politique, territorial et stratégique. De fait, ce livre volumineux mais bien écrit est organisé en trois parties, reprenant chaque aspect de manière chronologique. Essertel se base essentiellement sur les sources dites classiques telles que les archives écrites, tant publiées que manuscrites, mais - chose rare pour un historien - il prend aussi en 
compte les objets et leur collection comme témoins et assistants dans la reconstitution d'une géopolitique de l'évangélisation en Nouvelle-Zélande.

2 La première partie intitulée « Un homme en tension » analyse le rôle de Mgr Pompallier évoluant parmi plusieurs sphères : la romaine représentée par la Sacrée Congrégation de Propagation de la foi, celle de la congrégation incarnée par le supérieur de la Société de Marie et la sphère politique du gouvernement colonial britannique, d'autres puissances européennes et des chefferies locales des îles néo-zélandaises. Dans le premier chapitre, le lecteur découvre la réflexion de Jean-Baptiste Pompallier touchant aux qualités nécessaires à un missionnaire en Océanie. Il s'appuie sur son expérience et les circonstances et conclut qu'une recrue idéale devra, avant son départ, disposer au minimum d'un bagage linguistique tant en anglais qu'en maori et qu'il devra faire preuve des compétences nécessaires pour remplir les diverses fonctions au sein d'une organisation hiérarchisée. Malgré les efforts de Mgr Pompallier pour constituer une bonne équipe, l'immensité même du vicariat rend celui-ci ingérable et suscite dès 1840 les premières oppositions et critiques envers le vicaire. Le deuxième chapitre de cette section initiale étudie comment Pompallier tente de gérer la crise de confiance en rédigeant un règlement de vie adapté aux circonstances devant restaurer une vie spirituelle indispensable au moral et à l'efficacité de ses prêtres. En 1842, Pompallier découvre lors de son retour d'une longue tournée pastorale que son vicariat est au bord de la faillite et que tout le monde est d'accord sur un point : il est bien piètre administrateur ! La crise mène finalement à la constitution d'un vicariat d'Océanie centrale comprenant les îles Wallis, Futuna, Fiji, Tonga, Samoa et la Nouvelle-Calédonie placées sous l'égide de Pierre Bataillon, et aboutit à la diversification par le recrutement de prêtres séculiers ainsi qu'à l'internationalisation du clergé missionnaire avec un coadjuteur de locution anglaise. Ces développements sont approfondis dans le troisième chapitre. Mgr Pompallier, devenu évêque administrateur du diocèse d'Auckland en 1847, espère avec ces mesures mettre fin aux suspicions du gouvernement britannique à l'égard de la mission catholique française. Malgré la pédagogie déployée pour contrôler son clergé, des rumeurs circulent à propos des finances. Après son départ pour l'Europe en 1859 afin de rencontrer le pape et de trouver de nouvelles recrues, Pompallier se rend compte qu'il devra faire face à de nouvelles difficultés. Depuis le début de l'implantation du catholicisme, le clergé a été confronté non seulement aux pasteurs britanniques de la Church Missionary Society (implantée depuis 1814) et de la Wesleyan Missionary Society (implantée depuis 1822) mais aussi au gouvernement colonial britannique en la personne du Capitaine William Hobson, soit le "Consul Britannique et Vice-gouverneur de la Nouvelle-Zélande ». Le vicaire apostolique se voit presque malgré lui propulsé au milieu des événements locaux quand il exprime le souhait que la liberté religieuse - donc la possibilité d'être catholique - soit garantie lors de la signature du traité de Waitangi (6 février 1840) par lequel la Nouvelle-Zélande acquiert un statut de colonie britannique. Le bouc émissaire de la première guerre des terres qui éclate en mars 1845, est trouvé chez les catholiques et leur chef, l'évêque, l'Epikopo, qui est tenu responsable de l'agitation auprès des Māoris protestants. Les gouverneurs britanniques ne veulent pas admettre que les Māoris mettent en cause le traité de Waitangi et la question de la souveraineté des terres. Le dernier chapitre de la première partie détaille les guerres des terres de 1850 à 1862 et de 1862 à 1866 . Pendant toute cette période qui commence par le traité de Waitangi, l'Eglise catholique, à travers son évêque et ses prêtres, est restée constamment auprès des Māoris, tout en tolérant la colonisation britannique. 
L'Epikopo incarne ainsi un grand chef qui dérange le gouvernement colonial et les Églises protestantes.

3 La question du développement territorial est étayée dans la deuxième partie, plus concise et intitulée «Du vicariat d'Océanie occidentale à l'évêché d'Auckland. Géostratégie de la construction et du développement ". Après le débarquement à Hokianga en 1838, Pompallier s'installe immédiatement à Kororareka (Russell) où le quartier général de la mission devient un centre attirant pour les Maoris. Dès le début, Pompallier vise à étendre les postes de mission à l'échelle du pays. En 1845, le vicariat compte une bonne douzaine de stations dans l'île du Nord, mais échoue dans l'île du Sud. Le deuxième chapitre explique comment la mission acquiert la Sancta Maria, une goélette qui facilite grandement la tâche des tournées pastorales entreprises par la mission en Nouvelle-Zélande, Wallis et Futuna, Fidji et Tonga. Le troisième chapitre étudie les difficultés financières sous l'angle de l'implantation dans cet immense territoire. La communication par bateau et courrier est facilitée par la division en deux diocèses. Les Sœurs de la Mercy contribuent à la présence sur le territoire, surtout en matière d'éducation et de l'insertion sociale du catholicisme. Essertel termine la deuxième section du livre sur l'idée qu'avec la création d'un évêché, la mission commence à sortir des difficultés financières. Par contre, les guerres, les violences, les destructions de stations, la perte des recettes locales ruinent l'administration Pompallier.

4 La troisième partie intitulée « Stratégie, pédagogie et impact de l'évangélisation. Pour une perspective anthropologique» vise à examiner la stratégie d'évangélisation. De fait, l'historien Essertel entre réellement dans le domaine de l'anthropologie et de la théologie. Cette section se penche sur la question peu étudiée du processus d'évangélisation. Mgr Pompallier est relativement bien accueilli et est très vite perçu comme thaumaturge, un grand chef religieux au mana puissant. Il se doit maintenant de s'implanter solidement en Nouvelle-Zélande. Dans le deuxième chapitre, Essertel tire l'attention sur un questionnaire longtemps ignoré qui vise à obtenir des informations précises sur les peuples évangélisés et qui est primordial pour comprendre l'approche ethnographique des cultures par les missionnaires. JeanBaptiste Pompallier et ses prêtres participent aussi à cette collecte d'informations ethnographiques avec un discours qui oscille entre sympathie, dégoût et empathie, tout en respectant la théologie paulienne caractérisée par une volonté de vivre avec les Māoris et de se mêler à eux. Essertel explique dans le troisième chapitre comment le vicaire apostolique se voit acculé à faire le tri entre les " mauvais " (la superstition, l'impudeur, choses non conformes à la loi de la charité) et les " bons » aspects (la vie quotidienne, l'expression artistique, le kava, le mode vestimentaire et l'organisation sociale) de la culture maorie. Il est aidé dans sa démarche par la beauté et le faste de la messe catholique, mais aussi par des catéchistes maoris qui accompagnent Pompallier dans toutes ses visites aux villages māoris. Les catholiques n'exploitent que tardivement l'engouement pour le livre qui existe chez les Māoris car la presse catholique ne fut installée qu'en 1842. Dans le quatrième chapitre, l'auteur explique que l'évêque arrive à s'implanter solidement malgré la présence d'un christianisme multiforme et concurrentiel doublé de la présence coloniale britannique. Il endosse à merveille son rôle de grand chef au mana puissant. Essertel tente d'expliquer comment cette nouvelle religion qui vient bouleverser leur monde est perçue par les Māoris. Il insiste sur le fait que les Māoris sont des agents actifs dans tout le processus visant à comprendre les différences entre les diverses formes du christianisme. La généalogie 
(whakapapa - qu'Essertel écrit wakapapa) divine du christianisme catholique donne à ce dernier un avantage certain tout comme d'ailleurs le fait que les catholiques n'ayant ni femme ni enfants, n'achètent pas à l'instar des protestants, des terres pour subvenir aux besoins de leur famille. Avoir un prêtre dans la communauté devenait pour certaines tribus, synonyme d'accès au mana prestigieux puisqu'il appartenait à l'Epikopo. Le succès dépendait fortement du caractère et des capacités du missionnaire à s'insérer dans la vie quotidienne des Maoris. Le dernier chapitre du livre traite des témoins tangibles du processus d'évangélisation et du degré d'insertion du vicaire apostolique. Il suit pour cela la notion de "biographie des objets " et trace le chemin d'une douzaine d'objets océaniens qui constituent la collection Pompallier du moment de leur collecte à leur arrivée au dépôt du musée des Confluences de Lyon. Essertel étudie aussi des objets d'art. Il examine les représentations faites de l'évêque Pompallier, le Pīhopa Pomapārie. Celles-ci témoignent de la vision que les Māoris ont de lui en tant que chef maori et chef spirituel. Finalement, le retour en NouvelleZélande des restes de Jean-Baptiste Pompallier en 2002 atteste de la présence " charnelle » du père spirituel et fondateur du catholicisme au pays du long nuage blanc, Aotearoa.

5 Yannick Essertel conclut sa biographie détaillée en affirmant qu'il existait une géopolitique de l'évangélisation déterminée par l'immensité du territoire et l'ébranlement du milieu socio-culturel et géopolitique néo-zélandais. Dans cet environnement en pleine mutation culturelle et sociale et en tant que Français dans une colonie britannique, Pompallier insistait sur la dimension catholique, il développa une stratégie de communication en langue maorie, et investit dans la politique d'insertion. Les annexes élaborées et tout aussi minutieuses que le livre même sont composées (1) de repères chronologiques, (2) d'un texte de Pompallier sur les productions de la Nouvelle-Zélande et les mœurs des Māoris, (3) du traité de Waitangi en anglais et en "néo-zélandais ", (4) de la première leçon de catéchisme du vicaire apostolique dans une tribu, (5) d'une lettre de Petit-Jean sur l'esprit du vrai missionnaire, (6) d'un poème de Pompallier, (7) d'une lettre pastorale, carême 1851, (8) d'une lettre de Pompallier au second roi maori, (9) d'une lettre pastorale de Pompallier sur les effets de la guerre, (10) du document de la liquidation du contentieux de l'administration Pompallier, (11) d'une carte avec les stations les plus importantes de la mission catholique en Nouvelle-Zélande (1838-1845), (12) de sigles et d'abréviations, et d'un (13) lexique. La bibliographie est organisée par thème, ce qui donne au lecteur intéressé un moyen facile d'approfondir un sujet ou aspect particulier qui relève de l'évangélisation en Océanie.

6 Dans cette œuvre si détaillée et approfondie, l'orthographe surprenante de certains mots maoris (comme wakapapa pour whakapapa, ou wakapakoko pour whakapakoko), l'usage d'une orthographe ancienne et francisée de noms de lieu dans le Pacifique (par example Béa pour Pea) et l'application du terme "néo-zélandais " pour désigner les Maoris ou leur langue, le maori, ne réduisent en rien les efforts fournis par Yannick Essertel. Les huit ans de travail, de fouilles dans les archives et collections de musées ont abouti à un livre absolument passionnant qui se lit aisément, mais qui par-dessus tout donne un portrait rigoureux de la vie missionnaire du premier évêque catholique en Nouvelle-Zélande. Parmi le nombre croissant de livres qui étudient l'impact du christianisme en Océanie cet ouvrage prend une place unique tant par sa focalisation autour d'un personnage que par les contextes géopolitique et culturel esquissés. 


\section{AUTEUR}

\section{FANNY WONU VEYS}

Nationaal Museum van Wereldculturen, Pays-Bas 